\author{
Ecología
}

\title{
Variación estacional de la herpetofauna en el cerro del Veinte, Irapuato, Guanajuato, México
}

\author{
Seasonal variation of the herpetofauna at the Cerro del Veinte, Irapuato, Guanajuato, Mexico \\ Adrian Leyte-Manrique ${ }^{\mathrm{a}, *}$, Juan Pablo Morales-Castorena ${ }^{\mathrm{b}}$ y Luis A. Escobedo-Morales ${ }^{\mathrm{b}}$ \\ a Laboratorio de Biología, Departamento de Innovación Agrícola Sustentable, Instituto Tecnológico Superior de Salvatierra, Manuel Gómez Morin 300, \\ Col. Janicho, 39800, Salvatierra, Guanajuato, México \\ ${ }^{\mathrm{b}}$ Estación Biológica, Instituto Tecnológico Superior de Irapuato, Carretera Irapuato-Silao, Km 12.5 s/n, Col. El Copal, 36821, Irapuato, Guanajuato, México \\ Recibido el 10 de febrero de 2015; aceptado el 7 de septiembre de 2015 \\ Disponible en Internet el 12 de febrero de 2016
}

\begin{abstract}
Resumen
En este estudio se analizó la diversidad de los anfibios y reptiles del cerro del Veinte, Irapuato, Guanajuato, para las estaciones de lluvias y secas en un ambiente de selva baja caducifolia. Se registraron 23 especies de herpetozoos, 6 anfibios y 17 reptiles, de las cuales 22 se presentaron en la temporada de lluvias y 12 en la seca. Anfibios como la rana Hyla eximia fueron abundantes en temporadas de lluvias, en tanto que el sapo Anaxyrus compactilis fue poco abundante tanto en la estación seca como en la de lluvias. Para los reptiles, las especies más abundantes en temporadas de lluvias fueron la lagartija de collar Sceloporus torquatus y la tortuga casquito Kinosternon integrum, y en temporadas seca, la lagartija espinosa S. spinosus. El presente trabajo contribuye al conocimiento sobre los patrones de diversidad en ambientes tropicales estacionales, y aporta información actual sobre la herpetofauna para el municipio de Irapuato, sentando precedentes para desarrollo de estudios ecológicos y planes de conservación para los anfibios y reptiles en el sitio.

Derechos Reservados ( 2015 Universidad Nacional Autónoma de México, Instituto de Biología. Este es un artículo de acceso abierto distribuido bajo los términos de la Licencia Creative Commons CC BY-NC-ND 4.0.
\end{abstract}

Palabras clave: Anfibios; Reptiles; Selva baja caducifolia; Estacionalidad

\section{Abstract}

In this study the diversity of amphibians and reptiles from the Cerro del Veinte, Irapuato, Guanajuato was analyzed and compared for rainy and dry seasons in a tropical dry forest. A total of 23 species were recorded, 6 amphibians and 17 reptiles of which 22 were collected in the rainy season and 12 in the dry season. The frog Hyla eximia was the most abundant amphibian species for the rainy season, while the toad Anaxyrus compactilis showed low abundance in both, dry and rainy seasons. For reptiles, the most abundant species during the rainy season were the collared lizard Sceloporus torquatus, and the turtle Kinosternon integrum, and in dry weather, the spiny lizard S. spinosus. This work contributes to the understanding of diversity patterns in seasonal tropical environments providing current information on the herpetofauna for the municipality of Irapuato, laying basis for the development of ecological studies and conservation plans for amphibians and reptiles on the site.

All Rights Reserved (C) 2015 Universidad Nacional Autónoma de México, Instituto de Biología. This is an open access item distributed under the Creative Commons CC License BY-NC-ND 4.0.

Keywords: Amphibians; Reptiles; Tropical deciduous forest; Seasonality

\footnotetext{
* Autor para correspondencia.

Correo electrónico: aleyteman@gmail.com (A. Leyte-Manrique).

La revisión por pares es responsabilidad de la Universidad Nacional Autónoma de México.
}

\section{Introducción}

La topografía y orografía de México hacen posible la heterogeneidad de paisajes y ecosistemas que lo caracterizan y que ha dado como resultado la alta diversidad florística y faunística que le confieren el título de megadiverso (Flores-Villela y CansecoMárquez, 2004; Flores-Villela y Gerez, 1994; Ochoa-Ochoa 
y Flores-Villela, 2006; Ramírez-Bautista, Hernández-Salinas, García-Vázquez, Leyte-Manrique y Canseco-Márquez, 2009). La diversidad de especies de vertebrados como los anfibios y reptiles es un ejemplo de ello, reconociéndose a la fecha para ambos grupos un total de 1,240 especies (376 anfibios y 864 reptiles; Flores-Villela y García-Vázquez, 2014; ParraOlea, Flores-Villela y Mendoza-Almeralla, 2014). Bajo este contexto, la herpetofauna en Guanajuato está representada por \pm 56 especies - 13 anfibios y 43 reptiles-, lo que corresponde al $4.51 \%$ de la herpetofauna del país (Flores-Villela y García-Vázquez, 2014; Parra-Olea et al., 2014). Sin embargo, Reynoso, González-Hernández y Sánchez-Luna (2012) mencionan un total de 102 especies - 25 anfibios y 77 reptiles - para el estado. Tal discrepancia en el número de especies para la entidad pone en evidencia la necesidad de trabajos herpetofaunísticos en Guanajuato (Flores-Villela y García-Vázquez, 2014; Campos-Rodríguez et al., 2009).

En municipios como Irapuato el conocimiento de la herpetofauna es casi nulo, teniéndose a la fecha únicamente el trabajo de Uriarte-Garzón y Lozoya-Gloria (2009) en el Área Natural Protegida Cerro de Arandas. Estos autores registraron un total de 20 especies - 5 anfibios y 15 reptiles-. En este contexto, los ambientes tropicales estacionales presentan variaciones de temperatura, humedad y de precipitación que influyen en la disponibilidad de recursos como el alimento y el microhábitat, lo que se refleja en fluctuaciones en la estructura, composición y diversidad de las comunidades de anfibios y reptiles presentes en estos ambientes (García y Cabrera-Reyes, 2008; Vitt y Caldwell, 2014; Zug, Vitt y Caldwell, 2001). El objetivo de este trabajo fue conocer y evaluar la diversidad y composición estacional de los herpetozoos en un ambiente de selva baja caducifolia del municipio de Irapuato. Se espera que los resultados de este trabajo contribuyan a incrementar el conocimiento de la diversidad herpetofaunística y den pauta para la generación de estudios futuros en ecología, así como para el planteamiento de estrategias de conservación ad hoc para los anfibios y reptiles del municipio de Irapuato y de la región.

\section{Materiales y métodos}

El cerro del Veinte se encuentra localizado en la región del Bajío, en el centro del estado de Guanajuato, y comprende una superficie aproximada de 6,120.52 ha (fig. 1). La elevación oscila entre los 1,770 y $2,340 \mathrm{~m}$ snm, el tipo de clima es semicálidosubhúmedo con un régimen de lluvias en verano, conforme a la clasificación climática de Köppen, modificada por García (1973). El tipo de vegetación característico en el sitio corresponde a la selva baja caducifolia (Rzedowski, 1978; Rzedowski y Calderón de Rzedowski, 1987). La temperatura media anual oscila entre 16 y $28^{\circ} \mathrm{C}$, siendo el mes más cálido mayo, con una temperatura promedio de $28^{\circ} \mathrm{C}$, y el más frío enero, con una temperatura promedio de $16^{\circ} \mathrm{C}$. La precipitación media anual es de $700 \mathrm{~mm}$, siendo el mes más lluvioso agosto, con un valor promedio anual de $1,700 \mathrm{~mm}$, y el mes con menor precipitación, febrero, con $300 \mathrm{~mm}$ (García, 1973).

El trabajo de campo en el cerro del Veinte se desarrolló de junio de 2012 a mayo de 2013 durante las estaciones de lluvias
- junio-septiembre- y secas - octubre-mayo-. Se llevaron a cabo 12 muestreos con una duración de 3 días cada uno y un esfuerzo de muestreo horas/hombre de $8 / 3=24 \mathrm{~h}$ (Moreno, 2001). El método de muestreó que se estableció fue por transectos de $1 \mathrm{~km}$ de largo por $10 \mathrm{~m}$ de ancho con 3 réplicas dentro de una matriz homogénea de vegetación de selva baja caducifolia (Feinsinger, 2003; Vite-Silva, Ramírez-Bautista y HernándezSalinas, 2010).

Para la recolecta de los anfibios y reptiles se emplearon técnicas convencionales de captura sugeridas por Casas-Andreu, Valenzuela-López y Ramírez-Bautista (1991), considerando para ello, además, la actividad y hábitos de los organismos. Anfibios: debido a sus hábitos principalmente nocturnos, se buscaron de 19:00 a 12:00 h en cuerpos de agua y entre la vegetación adyacente. Se capturaron directamente con la mano y con ayuda de redes tipo entomológicas. Reptiles: se recolectaron de 8:00 a 17:00 h, dada su actividad predominantemente diurna, se buscaron en todos los microhábitats posibles como son rocas, troncos, arbustos, entre otros (Uribe-Peña, Ramírez-Bautista y CasasAndreu, 1999). La captura de lagartijas y tortugas se hizo con la mano y solo en caso de especies de lagartijas evasivas se emplearon ligas de plástico para inmovilizarlas. Las serpientes venenosas fueron recolectadas con ayuda de ganchos o pinzas herpetológicas, en tanto que las culebras se recolectaron directamente con la mano.

Los ejemplares capturados en campo se identificaron in situ a nivel de especie con ayuda de guías especializadas; por ejemplo, Ramírez-Bautista et al. (2009). Los ejemplares que no fueron identificados en campo se trasladaron al Laboratorio de Biología del Instituto Tecnológico Superior de Salvatierra para ser determinados con el apoyo de claves para grupos específicos de anfibios y reptiles. Los nombres científicos de las especies se asignaron de acuerdo con lo recomendado por Flores-Villela y Canseco-Márquez (2004) y Liner (2007). La captura, manejo y liberación de los organismos en campo se hizo bajo previa autorización del permiso de recolecta científica SGPA/DGVS/09749/2012 otorgado por la Dirección General de Vida Silvestre de la Semarnat.

\section{Análisis de datos}

Se realizó una curva de acumulación de especies a partir de los registros obtenidos en campo para determinar la representatividad del inventario herpetofaunístico (Halffter, Moreno y Pineda, 2001; Moreno, 2001; Moreno y Halffter, 2000). Se emplearon los estimadores no paramétricos Chao 1 y ACE, debido a que estos se basan en la abundancia de individuos de cada especie de una muestra representativa (Halffter et al., 2001; Moreno y Halffter, 2000). Las estimaciones fueron realizadas con el programa EstimateS Versión 7.5.0 (Colwell, 2006).

Se determinó la diversidad de especies de anfibios y reptiles por estación (lluvias y secas), mediante el índice de equidad de Shannon-Wiener, el cual asume la uniformidad de los valores de importancia a través de todas las especies de la muestra y mide el grado promedio de incertidumbre en la predicción de la pertenencia de un individuo a una especie en particular (Moreno, 2001; Vellend, 2001). Se aplicó una prueba no paramétrica de U 

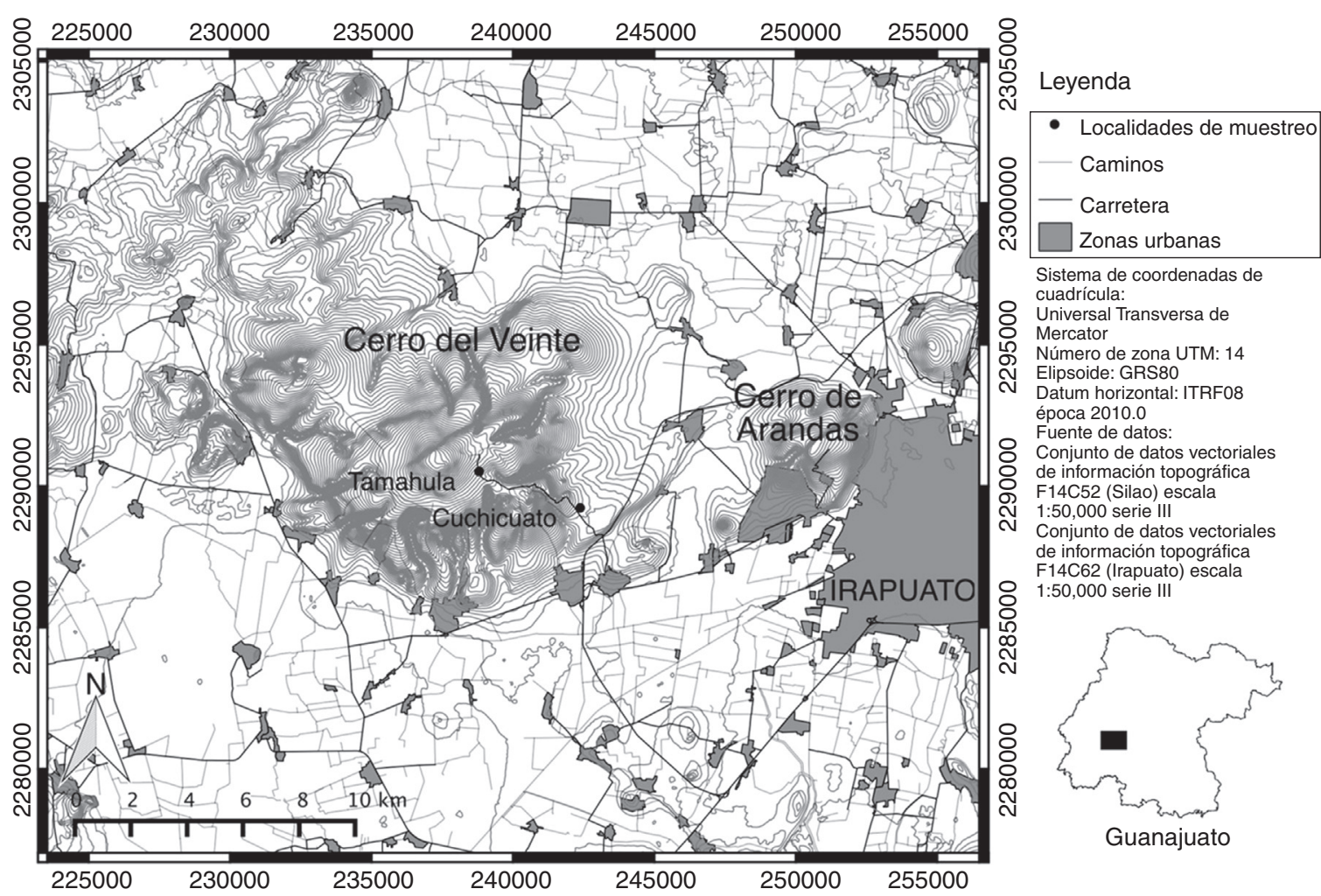

Figura 1. Ubicación geográfica del cerro del Veinte, municipio de Irapuato, Guanajuato.

Mann-Whitney para determinar si existían diferencias entre estaciones en cuanto a su diversidad. Para comparar gráficamente los patrones de abundancia de especies entre las estaciones de lluvias y secas se elaboraron curvas de Whittaker o de rangoabundancia, indicando la abundancia relativa de las especies registradas por estación. El programa utilizado para estos análisis fue BioDiversity Professional Versión 2 (Magurran, 2004).

\section{Resultados}

En este trabajo se registraron 23 especies de herpetozoos -6 anfibios y 17 reptiles-. Los anfibios estuvieron representados por los anuros con 6 especies, siendo la familia Hylidae la de mayor diversidad con 3 especies. Para los reptiles, el orden con mayor número de especies fue el de las serpientes con 11, seguido de los lacertilios con 5, y las tortugas con una sola especie (tabla 1). En cuanto a la riqueza por estación en la temporada de lluvias se presentaron 22 especies -6 de anfibios y 16 de reptiles-y en secas, 12 - una de anfibio y 11 de reptiles; tabla 2 -

Tabla 1

Diversidad de la herpetofauna en el cerro del Veinte, Irapuato, Guanajuato.

\begin{tabular}{llccc}
\hline Clase & Orden & Familias & Géneros & Especies \\
\hline Amphibia & Anura & 4 & 4 & 6 \\
Reptilia & Lacertilia & 2 & 2 & 5 \\
& Serpentes & 4 & 7 & 11 \\
& Testudines & 1 & 1 & 1 \\
Total & & 11 & 14 & 23 \\
\hline
\end{tabular}

El estimador Chao 1 predijo 24 especies - con un $85 \%$ de la completitud-y ACE, 27 especies -con una completitud del $96 \%$ - en tanto que la observada fue de 23 especies (fig. 2). La diversidad calculada para el índice de Shannon-Wiener arrojó un valor de $H^{\prime}=2.7$ para lluvias y de $H^{\prime}=1.9$ en secas, siendo estadísticamente significativa la diferencia entre estaciones (U Mann-Whitney, $U=1.2 \pm 1.6, P=0.0018$; fig. 3).

Las curvas de rango-abundancia o de Whittaker mostraron que la rana Hyla eximia fue la especie de anfibio más abundante en lluvias y Anaxyrus compactilis en secas. Para los reptiles, la lagartija Sceloporus torquatus y la tortuga Kinosternon integrum fueron abundantes en lluvias, y para secas las lagartijas $S$. spinosus y Aspidoscelis gularis. En serpientes tanto para la temporada de lluvias como en secas su abundancia fue baja (fig. 4).

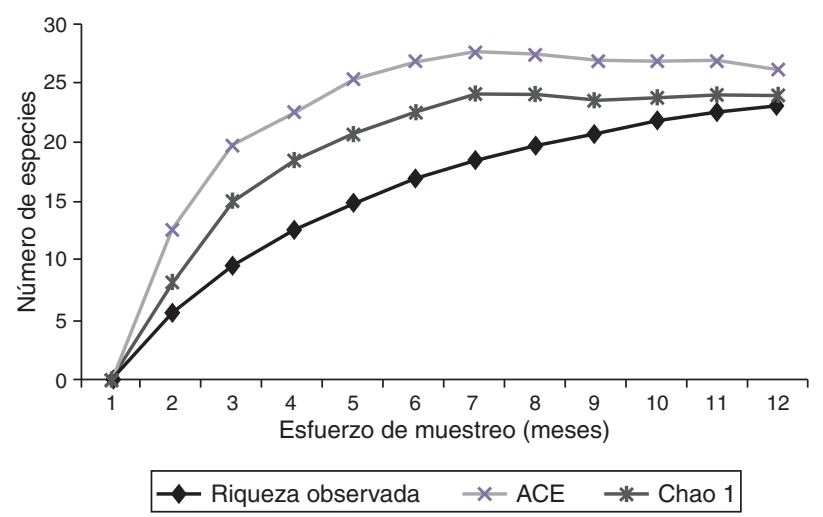

Figura 2. Curvas de acumulación de especies para la herpetofauna del cerro del Veinte, Irapuato, Guanajuato. 
Tabla 2

Especies de anfibios y reptiles registradas para el cerro del Veinte, Irapuato, Guanajuato. Se incluye su abundancia para cada estación (lluvias y secas) y su estatus conforme a la Nom-059-Semarnat-2010.

\begin{tabular}{|c|c|c|c|c|}
\hline Taxa & * & Lluvias & Secas & Estatus \\
\hline Clase Amphibia Anura & & & & $\mathrm{Nc}$ \\
\hline \multicolumn{5}{|l|}{ Familia Bufonidae } \\
\hline Anaxyrus compactilis (Wiegmann, 1833) & I & 2 & 1 & $\mathrm{Nc}$ \\
\hline \multicolumn{5}{|l|}{ Craugastoridae } \\
\hline Craugastor augusti (Dugés, 1879) & $\mathrm{D}$ & 3 & & $\mathrm{Nc}$ \\
\hline \multicolumn{5}{|l|}{ Hylidae } \\
\hline Hyla arenicolor Cope, 1866 & $\mathrm{~J}$ & 2 & & $\mathrm{Nc}$ \\
\hline Hyla eximia Baird, 1864 & $\mathrm{C}$ & 5 & & $\mathrm{Nc}$ \\
\hline Hyla plicata Brocchi, 1877 & $\mathrm{E}$ & 3 & & A \\
\hline \multicolumn{5}{|l|}{ Scaphiopodidae } \\
\hline Spea multiplicata (Cope, 1863) & $\mathrm{K}$ & 2 & & $\mathrm{Nc}$ \\
\hline \multicolumn{5}{|l|}{ Clase Reptilia Lacertilia } \\
\hline \multicolumn{5}{|l|}{ Familia Phrynosomatidae } \\
\hline Sceloporus grammicus Wiegmann, 1828 & $\mathrm{~F}$ & 3 & 1 & $\operatorname{Pr}$ \\
\hline Sceloporus scalaris Wiegmann, 1828 & $\mathrm{~L}$ & 2 & 1 & $\mathrm{Nc}$ \\
\hline Sceloporus spinosus Wiegmann, 1828 & G & 3 & 8 & $\mathrm{Nc}$ \\
\hline Sceloporus torquatus Wiegmann, 1828 & A & 15 & 2 & $\mathrm{Nc}$ \\
\hline \multicolumn{5}{|l|}{ Teiidae } \\
\hline Aspidoscelis gularis (Baird y Girard, 1852) & $\mathrm{P}$ & 1 & 8 & $\mathrm{Nc}$ \\
\hline \multicolumn{5}{|l|}{ Serpente/Colubridae } \\
\hline Coluber mentovarius (Mertens, 1934) & Q & 1 & 1 & A \\
\hline Conopsis lineata (Kennicott, 1859) & M & 2 & & $\mathrm{Nc}$ \\
\hline Conopsis nasus Günther, 1858 & $\mathrm{H}$ & 3 & & $\mathrm{Nc}$ \\
\hline Conopsis sp & $\mathrm{R}$ & 1 & & $\mathrm{Nc}$ \\
\hline Pituophis deppei (Duméril, 1853) & S & 1 & 1 & A \\
\hline Salvadora bairdi (Jan, 1860) & $\mathrm{N}$ & 2 & 1 & $\operatorname{Pr}$ \\
\hline Senticolis triaspis (Cope, 1866) & $\mathrm{V}$ & & 1 & $\mathrm{Nc}$ \\
\hline \multicolumn{5}{|l|}{ Elapidae } \\
\hline Micrurus tener (Baird y Girard, 1853) & $\mathrm{T}$ & 1 & & $\operatorname{Pr}$ \\
\hline \multicolumn{5}{|l|}{ Typhlopidae } \\
\hline Indotyphlops braminus (Daudin, 1803) & $\tilde{\mathrm{N}}$ & 2 & & $\mathrm{Nc}$ \\
\hline \multicolumn{5}{|l|}{ Viperidae } \\
\hline Crotalus aquilus Klauber, 1952 & $\mathrm{U}$ & 1 & 1 & $\operatorname{Pr}$ \\
\hline Crotalus molossus Baird y Girard, 1853 & $\mathrm{O}$ & 2 & 1 & $\operatorname{Pr}$ \\
\hline \multicolumn{5}{|l|}{ Testudines/Kinosternidae } \\
\hline Kinosternon integrum Le Conte, 1824 & $\mathrm{~B}$ & 6 & & $\operatorname{Pr}$ \\
\hline Total & & 46 & 27 & \\
\hline
\end{tabular}

A: amenazada; E: probablemente extinta en el medio silvestre; Nc: no considerada; P: peligro de extinción; Pr: protección especial.

* Las letras en mayúsculas representan la abundancia y ubicación de cada especie en la figura 4.

\section{Discusión}

En el cerro del Veinte se registró una riqueza de 23 especies -6 anfibios y 17 reptiles-. Los estimadores no paramétricos utilizados para generar las curvas de acumulación de especies Chao 1 y ACE predijeron un total de 24 y 27, es decir, el 85 y $96 \%$ de la completitud del inventario, respectivamente. Sin embargo, la riqueza observada en la gráfica no alcanza la asíntota, sugiriendo con ello que un mayor número de especies podría registrarse en el área (fig. 2).

La diversidad expresada mediante el índice de ShannonWiener por estación del año fue mayor en lluvias (fig. 3), patrón ya señalado en otros estudios en ambientes tropicales estacionales (Vite-Silva et al., 2010). Al respecto Huey et al. (2009) señalan que tanto anfibios como reptiles son sensibles a temperaturas elevadas durante la temporada de secas, la cual conduce a una reducción de su actividad que influye en patrones de riqueza, abundancia y distribución observados tanto local como estacionalmente (Orea-Gadea, 2010; Uriarte-Garzón y Lozoya-Gloria, 2009; Vitt y Caldwell, 2014). La baja diversidad de especies de anfibios en el cerro del Veinte con respecto a ambientes no estacionales con mayor humedad como son selvas perennifolias y bosques mesófilos de montaña podría ser una respuesta directa al estrés fisiológico ocasionado por las condiciones ambientales imperantes en selvas bajas caducifolias a lo largo del año, como son las bajas precipitaciones y escasez 


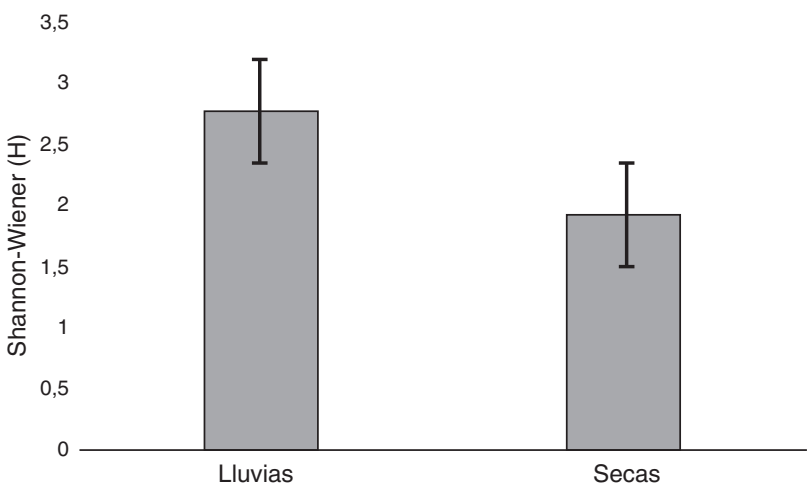

Figura 3. Índice de Shannon-Wiener estimado para las estaciones de lluvias y secas de la herpetofauna presente en el cerro del Veinte, Irapuato, Guanajuato.

de cuerpos de agua permanentes que influyen en su actividad reproductiva, su abundancia y diversidad (Heyer, Donnelly, Mcdiarmid, Hayek y Foster, 1994; García y Cabrera-Reyes, 2008; Luria-Manzano, 2012; ver figura 4). A diferencia de los anfibios, los reptiles en el cerro del Veinte se registraron en las 2 estaciones del año. Esto sugiere la ventaja de adaptaciones fisiológicas, morfológicas, reproductivas y conductuales para hacer frente a las variaciones climáticas y de temperatura que son comunes en ambientes de tipo estacional en los que, además, ocurren cambios temporales en la composición de clases de edad y abundancia de las especies (Cruz-Elizalde y Ramírez-Bautista, 2012; Ramírez-Bautista y Moreno, 2006; Vite-Silva et al., 2010; Vitt y Caldwell, 2014).

Las curvas de rango-abundancia mostraron que la composición y abundancia de especies entre estaciones difirió - pocas especies abundantes y muchas raras; fig. 4-. Al respecto, Berriozábal-Islas (2012) y Hernández-Salinas (2009)

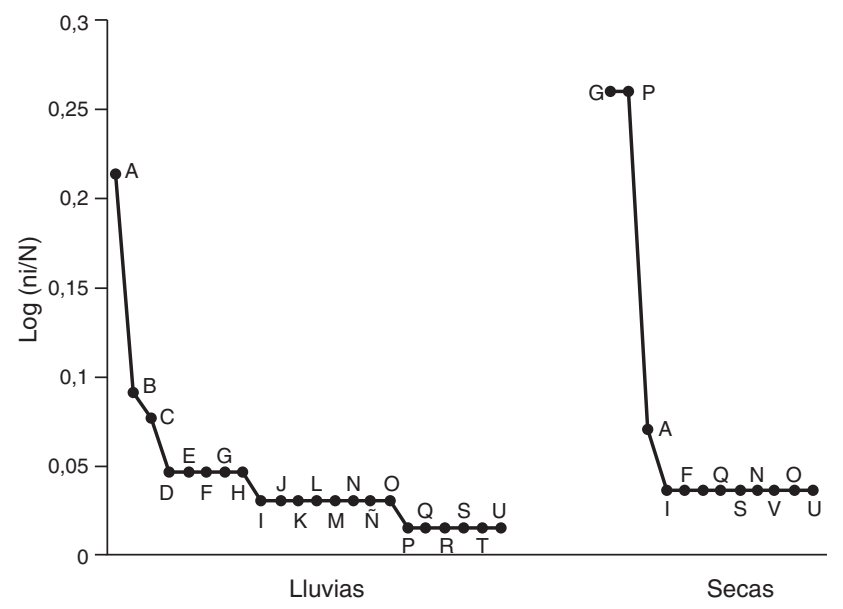

Figura 4. Curvas de rango-abundancia o de Whittaker. Muestran la abundancia de las especies de anfibios y reptiles para las estaciones de lluvias y secas en el cerro del Veinte, Irapuato, Guanajuato. Las letras representan a las especies: $\mathrm{A}=$ Sceloporus torquatus, $\mathrm{B}=$ Kinosternon integrum, $\mathrm{C}=$ Hyla eximia, $\mathrm{D}=$ Craugastor augusti, $\mathrm{E}=$ Hyla plicata, $\mathrm{F}=$ Sceloporus grammicus, $\mathrm{G}=$ Sceloporus spinosus, $\mathrm{H}=$ Conopsis nasus, $\mathrm{I}=$ Anaxyrus compactilis, $\mathrm{J}=$ Hyla arenicolor, $\mathrm{K}=$ Spea multiplicata, $\mathrm{L}=$ Sceloporus scalaris, $\mathrm{M}=$ Conopsis lineata, $\mathrm{N}=$ Salvadora bairdi, $\tilde{\mathrm{N}}=$ Indotyphlops braminus, $\mathrm{O}=$ Crotalus molossus, $\mathrm{P}=$ Aspidoscelis gularis, $\mathrm{Q}=$ Coluber mentovarius, $\mathrm{R}=$ Conopsis $\mathrm{sp}, \mathrm{S}=$ Pituophis deppei, $\mathrm{T}=$ Micrurus tener, $\mathrm{U}=$ Crotalus aquilus, $\mathrm{V}=$ Senticolis triaspis. consideran que el comportamiento de este tipo de curvas es debido a la presencia de especies raras cuya densidad poblacional suele ser baja y marcadamente influenciada por la variación en los ciclos reproductivos estacionales, o por la disponibilidad de alimento a lo largo del año (Macip-Ríos y Muñoz-Alonzo, 2008; Vitt y Caldwell, 2014). En este caso los anfibios como Hyla eximia en el cerro del Veinte tuvieron mayor presencia y abundancia en las pozas de agua temporales que hay en el sitio, lo cual coincidió con la temporada de lluvias. Para los reptiles, las especies de lagartijas como Sceloporus torquatus, S. spinosus y A. gularis - registradas durante todo el año- han sido documentadas en ambientes tropicales estacionales del estado de Hidalgo como especies comunes, dada su tolerancia a cambios drásticos en el ambiente y a su capacidad de explotar los recursos disponibles (Ramírez-Bautista et al., 2014; Vite-Silva et al., 2010). En el caso de las serpientes, estas mostraron baja abundancia en estaciones secas y alta en lluvias, lo que concuerda con otros trabajos en ambientes tropicales estacionales (García y Cabrera-Reyes, 2008 [Chamela, Jalisco]; Vite-Silva et al., 2010 [Barranca de Metztitlán, Hidalgo]) en los que se registra una mayor abundancia de individuos y presencia en ambas estaciones, por lo que se puede hablar de un patrón que también fue observado en el cerro del Veinte.

La lista de especies registradas en este estudio contribuye al incremento en el conocimiento sobre la herpetofauna del municipio de Irapuato y, en particular, de ambientes tropicales estacionales dentro del estado de Guanajuato. De las 23 especies que se registraron, 9 de ellas (Hyla plicata, Sceloporus grammicus, Coluber mentovarius, Pituophis deppei, Salvadora bairdi, Micrurus tener, Crotalus aquilus, C. molossus y Kinosternon integrum) se incluyen en la Nom-059-Semarnat-2010, una se encuentra enlistada como amenazada y 8 bajo protección especial (Semarnat, 2010; tabla 2). Conocer los patrones estacionales de diversidad de los herpetozoos en el cerro del Veinte puede permitir el planteamiento de estudios futuros dirigidos a aspectos de su historia natural, así como de conservación para estos vertebrados en el sitio.

\section{Agradecimientos}

Agradecemos a los habitantes de las comunidades de Tamaula y Cuchicuato en el cerro del Veinte, a E. M. Hernández-Navarro, H. Parra-González y Joel Servín por su apoyo durante el trabajo en campo, a la Semarnat por el permiso otorgado para la recolecta científica, así como a los revisores anónimos que con sus comentarios enriquecieron la calidad de este manuscrito.

\section{Referencias}

Berriozábal-Islas, C. (2012). Riqueza y diversidad herpetofaunística del bosque tropical, cafetales y potreros del municipio de Huehuetla, Hidalgo, México. Universidad Autónoma del Estado de Hidalgo. Tesis.

Campos-Rodríguez, J., Elizalde-Arellano, C., López-Vidal, J., AguilarMartínez, G., Ramos-Reyes, S. y Hernández-Arciga, R. (2009). Nuevos registros de anfibios y reptiles para Guanajuato, procedentes de la Reserva de la Biosfera «Sierra Gorda de Guanajuato» y zonas adyacentes. Acta Zoológica Mexicana, 25, 269-282. 
Casas-Andreu, G., Valenzuela-López, G. y Ramírez-Bautista, A. (1991). Cómo hacer una colección de anfibios y reptiles. México, D.F.: Cuadernos del Instituto de Biología, Universidad Nacional Autónoma de México.

Colwell, R.K. (2006). EstimateS: Statistical estimation of species richness and shared species from samples. Versión 8.2. Users guide and application. Recuperado el 14 de abril de 2014 de: http://purl.oclc.or/estimates.

Cruz-Elizalde, R. y Ramírez-Bautista, A. (2012). Diversidad de reptiles en tres tipos de vegetación del estado de Hidalgo. México. Revista Mexicana de Biodiversidad, 83, 458-467.

Feinsinger, P. (2003). El diseño de estudios de campo para la conservación de la biodiversidad. Santa Cruz de la Sierra, Bolivia: Editorial FAN.

Flores-Villela, O. A. y Canseco-Márquez, L. (2004). Nuevas especies y cambios taxonómicos para la herpetofauna de México. Acta Zoológica Mexicana, 20, $115-144$.

Flores-Villela, O. A. y García-Vázquez, U. (2014). Biodiversidad de reptiles en México. Revista Mexicana de Biodiversidad (Suplemento), 85, S467-S475.

Flores-Villela, O. A. y Gerez, P. (1994). Biodiversidad y conservación en México: vertebrados, vegetación y uso de suelo. México, D.F.: Comisión Nacional para el Conocimiento y Uso de la Biodiversidad y Universidad Nacional Autónoma de México.

García, E. (1973). Modificaciones al sistema de clasificación climática de Köppen: para adaptarlo a las condiciones de la República Mexicana. (Segunda edición). México, D.F.: Universidad Nacional Autónoma de México.

García, A. y Cabrera-Reyes, A. (2008). Estacionalidad y estructura de la vegetación en la comunidad de anfibios y reptiles de Chamela, Jalisco, México. Acta Zoológica Mexicana, 24, 91-115.

Halffter, G., Moreno, C.E. y Pineda, E.O. (2001). Manual para evaluación de la biodiversidad en reservas de la biosfera. Vol. 2. Zaragoza: MyT-Manuales y Tesis SEA.

Hernández-Salinas, U. (2009). Estudio herpetofaunístico del estado de Hidalgo, México. Tesis de maestría. Universidad Autónoma del Estado de Hidalgo.

Heyer, W. R., Donnelly, M. A., Mcdiarmid, R. W., Hayek, L. R. y Foster, M. S. (1994). Measuring and monitoring biological diversity: standard methods for amphibians. Washington D.C: Smithsonian Institution.

Huey, R. B., Deutsch, C. A., Tewksbury, J. J., Vitt, L. J., Hertz, P. E., ÁlvarezPérez, H. J., et al. (2009). Why tropical forest lizards are vulnerable to climate warming? Proceedings of the Royal Society B, Biological Sciences, 276, 1939-1948.

Liner, E. (2007). A checklist of the amphibians and reptiles of Mexico. Occasional Papers of the Museum of Natural Science, 80, 1-59.

Luria-Manzano, R. (2012). Ecología trófica del ensamble de anuros riparios de San Sebastián. Tlacotepec, Sierra Negra de Puebla, México. Tesis de maestría. Universidad Autónoma del Estado de Hidalgo.

Macip-Ríos, R. y Muñoz-Alonzo, A. (2008). Diversidad de lagartijas en cafetales y bosque primario en el Soconusco chiapaneco. Revista Mexicana de Biodiversidad, 79, 185-195.

Magurran, A. E. (2004). Measuring biological diversity. Oxford: Blackwell.

Moreno, C.E. (2001). Métodos para medir la biodiversidad. Vol. 1. Zaragoza: MyT-Manuales y Tesis SEA.

Moreno, C. E. y Halffter, G. (2000). Assessing the completeness of bat biodiversity inventories using species accumulation curves. Journal of Applied Ecology, 37, 149-158.
Ochoa-Ochoa, L. M. y Flores-Villela, O. A. (2006). Áreas de diversidad y endemismo de la herpetofauna mexicana. México, D.F.: Universidad Nacional Autónoma de México y Comisión Nacional para el Conocimiento y Uso de la Biodiversidad.

Orea-Gadea, J. (2010). Efecto de plantaciones mixtas sobre la diversidad herpetofaunística en selva baja caducifolia de Sierra de Huautla. Morelos: Tesis. Universidad Autónoma del Estado de Morelos.

Parra-Olea, G., Flores-Villela, O. y Mendoza-Almeralla, C. (2014). Biodiversidad de anfibios en México. Revista Mexicana de Biodiversidad Suplemento, 85, S460-S466

Ramírez-Bautista, A., Hernández-Salinas, U., Cruz-Elizalde, R., BerriozábalIslas, C., Lara-Tufiño, D., Goyenechea Mayer-Goyenechea, I., et al. (2014). Los anfibios y reptiles de Hidalgo, México: diversidad, biogeografía y conservación. Pachuca: Sociedad Herpetológica Mexicana, A.C.

Ramírez-Bautista, A., Hernández-Salinas, U., García-Vázquez, O., LeyteManrique, A. y Canseco-Márquez, L. (2009). Herpetofauna del Valle de México: diversidad y conservación. México, D.F.: Universidad Autónoma del Estado de Hidalgo y Comisión Nacional para el Conocimiento y Uso de la Biodiversidad.

Ramírez-Bautista, A. y Moreno, C. (2006). Análisis comparativo de la herpetofauna de cuatro regiones geográficas de México. En A. Ramírez-Bautista, L. Canseco-Márquez y F. Mendoza-Quijano (Eds.), Inventarios herpetofaunísticos de México: avances en el conocimiento de su biodiversidad 3 (pp. 74-98). México, D.F.: Publicaciones Sociedad Herpetológica Mexicana.

Reynoso, V.H., González-Hernández, A. y Sánchez-Luna, M. (2012). Anfibios y reptiles. En Conabio/IEE (Eds.), La biodiversidad en Guanajuato: estudio de estado Vol. II (pp. 220-226). México, D.F.: Comisión Nacional para el Conocimiento y Uso de la Biodiversidad e Instituto de Ecología del Gobierno del Estado de Guanajuato.

Rzedowski, J. (1978). Vegetación de México. México, D.F.: Limusa.

Rzedowski, J. y Calderón de Rzedowski, G. (1987). El bosque tropical caducifolio de la región mixteca del Bajío. Trace, 12, 12-21.

Semarnat (Secretaría de Medio Ambiente y Recursos Naturales). (2010). Norma Oficial Mexicana NOM-059-SEMARNAT-2010, Protección ambientalEspecies nativas de México de flora y fauna silvestres-Categorías de riesgo y especificaciones para su inclusión, exclusión o cambio-Lista de especies en riesgo. Diario Oficial de la Federación. 30 de diciembre de 2010, Segunda Sección. México.

Uriarte-Garzón, P. y Lozoya-Gloria, E. (2009). Manual del inventario de la fauna del Área Natural Protegida «Cerro de Arandas». Irapuato: Parque Ecológico de Irapuato, A.C.

Uribe-Peña, Z., Ramírez-Bautista, A. y Casas-Andreu, G. (1999). Anfibios y reptiles de las serranías del Distrito Federal. México, D.F.: Instituto de Biología, Universidad Nacional Autónoma de México. Cuadernos 32.

Vellend, M. (2001). Do commonly-used indices of B-diversity measure species turnover? Journal of Vegetation Science, 12, 545-552.

Vite-Silva, V., Ramírez-Bautista, A. y Hernández-Salinas, U. (2010). Diversidad de anfibios y reptiles de la Reserva de la Biosfera Barranca de Metztitlán, Hidalgo, México. Revista Mexicana de Biodiversidad, 81, 473-485.

Vitt, J. L. y Caldwell, J. P. (2014). Herpetology: An introduction biology of amphibians and reptiles (Cuarta edición). San Diego: Academic Press Elsevier.

Zug, G. R., Vitt, 1. J. y Caldwell, J. P. (2001). Herpetology: an introduction biology of amphibians and reptiles (Segunda edición). San Diego: Academic Press Elsevier. 